\title{
On Optimal Frequencies for Reconstruction of a One-Dimensional Profile of Gradient Layer's Refractive Index
}

\author{
Dmitrii Tumakov \\ Kazan Federal University, 18 Kremlyovskaya Street, Kazan 420008, Russia \\ Correspondence should be addressed to Dmitrii Tumakov; dtumakov@kpfu.ru
}

Received 26 May 2014; Revised 27 November 2014; Accepted 30 November 2014; Published 16 December 2014

Academic Editor: Stefan Wabnitz

Copyright (C) 2014 Dmitrii Tumakov. This is an open access article distributed under the Creative Commons Attribution License, which permits unrestricted use, distribution, and reproduction in any medium, provided the original work is properly cited.

\begin{abstract}
The problem of reconstruction of a one-dimensional profile of gradient layer's refractive index is investigated. An algorithm for choosing a right frequency, at which a scattered field is measured, is proposed. It is concluded that at the correct choice of frequency one measurement must be sufficient. Moreover, in this case, regularization parameters of the residual functional are chosen as zero. It is shown that in case of measurements being carried out with errors, residual terms must be added to the functional.
\end{abstract}

\section{Introduction}

For developing efficient antireflective coatings on the solid surfaces, the structure of coating layers often requires a change in physical properties of the layers across the direction of light propagation by setting a proper gradient in this direction. This engineering application has been under thorough investigation for quite a long time, for example, through studying evaporation of very thin alternating high/low refractive index films creating an effective refractive index gradient by varying thicknesses of the layers $[1,2]$.

For developing the right technology of manufacturing the coating layers and testing the manufactured layers as well as for other applications encountered in optics and electrodynamics, it is often required to reconstruct refractive indices of the layers. Thus, reconstruction of a one-dimensional profile of the layer's refractive index, which is an inverse problem, is one of the major problems in this field. Reconstruction of an unknown profile can be carried out using various types of data such as data for reflection coefficients, input impedance, and scattered electric or magnetic fields. In those techniques, the reconstructed profile can be considered as either a complex profile or a continuous profile [3].

For profile reconstructions, one of the following two approaches is usually utilized: time-domain methods $[4,5]$ or frequency-domain methods. The time-domain methods require a rather sophisticated and high-precision equipment for generating and registering narrow pulses, which complicates a practical application of these methods. Often the use is made of terahertz pulsed spectroscopy $[6,7]$. The frequency-domain methods are used for determining the desired parameters through measuring multiple frequencies, multiple angles, and different polarizations. The permittivity profile is either approximated by an expansion series involving a finite number of elements [8] or represented in the form of some discrete values [9].

The inverse problems are ill-posed problems, and regularization methods are often used to find a solution to these problems. Methods of this kind stabilize the solution but lead to certain losses of accuracy. Convergence of the frequencydomain methods depends on the choice of the frequency range used in the inverse problem. However, rigorous criteria for choosing the frequency range have not yet been proposed [10].

Among all the possible solution techniques, analytical approximation techniques [11-13] and layer stripping techniques $[14,15]$ can be especially emphasized. Most often, the profile reconstruction problem is solved by an optimization method that minimizes the error between the observed and calculated data. Besides, the problems, in which the data are either incomplete or contaminated with some noise, are also frequent [16].

The optimization methods can be further subdivided into local and global optimization methods. Examples of local 
optimization methods include gradient methods and quasiNewton and Gauss-Newton techniques $[17,18]$. These methods are fast but often converge to local minima due to the nonlinear nature of the problem. Therefore, these methods are recommended for being used only in case of availability of sufficient amount of priory information. Global optimization methods do not require any a priori information, but a large number of iterations are needed to reach convergence.

Of all the existing global optimization methods, which are used in electromagnetic inversion problems, the neural network technique [19], the genetic algorithms [20, 21], and the particle swarm optimization techniques [22-26] can be accentuated. Each of these methods has its own advantages and disadvantages [27-29]. In view of this, sometimes hybrid techniques combining different methods for their advantages are utilized [30, 31].

In the present work, an algorithm developed in [32] is developed further. The key component of the algorithm is determining an optimal frequencies range for the profile reconstruction. First, the flow of energy transited through the layer is measured. Next, a frequency, at which the flow has a minimum, is determined. Measurements are carried out both after the layer and before the layer. The first reason for that is that measurements at both ends provide more stability to the problem. Secondly, it must be kept in mind that in certain cases different profiles ensure identical fields at the layer exit [33], while reflected fields can differ. High precision measurements of the entire diffracted field at a chosen frequency (or a narrow frequency interval) are carried out at the next stage.

The reconstruction problem is reduced to the minimization of a certain functional. For minimizing the functional, the direct problem is solved iteratively. The algorithm for reconstruction of refractive index is validated through the experiments with several frequency domains. At first, the case in which refractive index of a layer monotonically increases and then monotonically decreases is considered. The layer having a complex profile is considered next. One of the sections is devoted to problems related to perturbed original measured data.

Gradient layers made up of different $\mathrm{TiO}_{2} / \mathrm{SiO}_{2}$ ratios are chosen for numerical experiments. Refractive indices of $\mathrm{TiO}_{2}$ and $\mathrm{SiO}_{2}$ are approximately 2.5 and 1.5 , respectively, and both materials are optically transparent in the visible and near-IR region [34]. By varying the $\mathrm{TiO}_{2} / \mathrm{SiO}_{2}$ ratios, thin films of any desired refractive index lying between those of $\mathrm{TiO}_{2}$ and $\mathrm{SiO}_{2}$ can be obtained. Refractive index of the composite material is determined by the $\mathrm{TiO}_{2} / \mathrm{SiO}_{2}$ ratio in a deposited dielectric film, defined as $\chi$ in $\left(\mathrm{TiO}_{2}\right)_{\chi}\left(\mathrm{SiO}_{2}\right)_{1-\chi}[35]$.

\section{Problem Statement}

Let the plane electromagnetic harmonic wave of type $u_{0}(x$, $t)=A_{0} \exp \left\{-i k_{0} n_{1} x+i \omega_{j} t\right\}$ fall on a layer of thickness $L$ with unknown refractive index $n_{2}(x)$ from a homogenous isotropic medium (see Figure 1), where $A_{0}$ is a wave amplitude, $k_{0}$ is the vacuum wave number, $n_{1}$ is the refractive index of medium 1 , and $\omega_{j}$ is a wave frequency. Variable $t$ is changed by $\omega$ and it is supposed that the functions depend on variables $x$ and $\omega$. It is necessary to reconstruct the refractive index

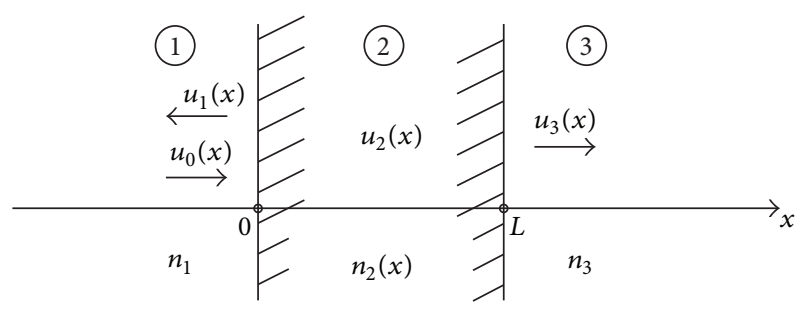

FIGURE 1: Geometry of the problem.

$n_{2}(x)$ by using the assumption that the reflected $u_{1}\left(x, \omega_{j}\right)$ and transmitted $u_{3}\left(x, \omega_{j}\right)$ waves are known for several frequencies $\omega_{j}$. The profile of refractive index $n_{2}(x)$ is supposed to vary continuously.

Measurements of $J$ different frequencies $\omega_{j}, j=1 \cdots J$, for intensity of magnetic and electric fields are already available; note that the measured values of intensities are real numbers. Thus, $J$ complex values of $u_{1}\left(0, \omega_{j}\right)$ and $u_{3}\left(L, \omega_{j}\right)$ are obtained (e.g., the values for TE-polarization represent intensity of the electric field) by the method of overdetermined boundary value problems [36].

So, $J$ sets of complex numbers $\widehat{u}_{0}^{j}=u_{0}\left(0, \omega_{j}\right)+u_{1}\left(0, \omega_{j}\right)$ are obtained for the values of the field in the layer at $x=0$ and $J$ sets of complex numbers $\widehat{u}_{L}^{j}=u_{3}\left(L, \omega_{j}\right)$ are obtained for the values of the field at $x=L$. In solving the direct problem of diffraction by a layer having refractive index $n_{2}(x), n_{2}(x)$ changes in such a way that the field acting as a solution to the direct problem provides values $\widehat{u}_{0}^{j}$ and $\widehat{u}_{L}^{j}$ on the layer boundaries.

\section{Direct Problem}

The direct problem for a harmonic wave of frequency $\omega_{j}$ will be solved [9]. In this section, $\omega_{j}$ will be fixed. Let the plane electromagnetic wave of type $u_{0}(x)=A_{0} \exp \left\{-i k_{0} n_{1} x\right\}$ fall on a layer of thickness $L$ with known refractive index $n_{2}(x)$ from a homogenous isotropic medium (see Figure 1). It is necessary to find the diffracted field or, in other words, the reflected, transited waves and a field excited in the layer $u_{2}(x)$.

The function $u(x)$ is continuous everywhere along with its derivatives and satisfies the equation

$$
u^{\prime \prime}(x)+k_{0}^{2} n^{2}(x) u(x)=0, \quad x \in \mathbf{R},
$$

where $n(x)$ is a known continuous function. For regions 1 and 3 of the medium, function $n(x)$ is constant and for these cases the equation is solved explicitly.

Thus, the diffraction problem is reduced to an ordinary differential equation

$$
u_{2}^{\prime \prime}(x)+k_{2}^{2}(x) u_{2}(x)=0, \quad 0<x<L,
$$

with boundary conditions

$$
\begin{gathered}
u_{2}^{\prime}(0)-i k_{1} u_{2}(0)=-2 i k_{1} A_{0}, \\
u_{2}^{\prime}(L)+i k_{3} u_{2}(L)=0,
\end{gathered}
$$


where $k_{j}=k_{0} n_{j}$ are wavenumbers of the media. The direct problem is solved numerically. The method of approximating an integral identity [37] is applied for increasing accuracy of the grid solution of the obtained boundary problem.

\section{Inverse Problem}

After $J$ measurements are carried out, the field components on the layer boundaries will be determined. The direct problem is solved iteratively for solving the inverse problem; the refractive index is updated using the value from the previous iteration. The initial approximation for $n_{2}(x)$ is a straight line connecting points $x=0$ and $x=L$ with values $n_{1}$ and $n_{3}$. If $n_{1}=n_{3}$, then the starting line is $n_{2}(x) \equiv n_{1}$. The functional defined by

$$
\begin{aligned}
F\left[n_{2}(x)\right]= & \sum_{j=1}^{J}\left|u_{2}^{j}(0)-\widehat{u}_{0}^{j}\right|^{2}+\sum_{j=1}^{J}\left|u_{2}^{j}(L)-\widehat{u}_{L}^{j}\right|^{2} \\
& +\alpha\left\|n_{2}(x)\right\|+\beta_{1}\left|n_{2}(0)-\widehat{n}_{0}\right|^{2} \\
& +\beta_{2}\left|n_{2}(L)-\widehat{n}_{L}\right|^{2}
\end{aligned}
$$

will be minimized, where $\widehat{u}_{0}^{j}$ and $\widehat{u}_{L}^{j}$ are known values of the measured field for the $j$ th experiment and $u_{2}^{j}(0)$ and $u_{2}^{j}(L)$ are the calculated values of the field at the current iterative step on both sides of the layer (at $x=0$ and $x=L$ ). $\alpha, \beta_{1}$, and $\beta_{2}$ are regularization parameters; $\widehat{n}_{0}$ and $\widehat{n}_{L}$ are known values of refractive index on the layer boundaries.

The function $n_{2}(x)$ is replaced with the vector $\mathbf{n}=\left\{n_{i}, i=\right.$ $0 \cdots N\}$. The new functional becomes

$$
\begin{aligned}
F[\mathbf{n}]= & \sum_{j=1}^{J}\left|u_{0}^{j}-\widehat{u}_{0}^{j}\right|^{2}+\sum_{j=1}^{J}\left|u_{N}^{j}-\widehat{u}_{L}^{j}\right|^{2}+\alpha \sum_{i=0}^{N}\left|n_{i}\right|^{2} \\
& +\beta_{1}\left|n_{0}-\widehat{n}_{0}\right|^{2}+\beta_{2}\left|n_{N}-\widehat{n}_{L}\right|^{2} .
\end{aligned}
$$

The method of golden section is used for minimization of the functional. The next condition imposed on $n_{i}$ is $n_{\min } \leq n_{i} \leq$ $n_{\max }$.

The relative error of the determined refractive index $\left\{n_{i}\right\}$ is

$$
\varepsilon=\frac{\sum_{j=0}^{N}\left|n_{2}\left(x_{i}\right)-n_{i}\right|^{2}}{\sum_{j=0}^{N}\left|n_{2}\left(x_{i}\right)\right|^{2}} .
$$

It can be noted that for a starting profile it is more convenient to choose a line connecting values closest to $n_{2}(0)$ and to $n_{2}(L)$ instead of using an interval connecting $n_{1}$ to $n_{3}$.

\section{Reconstruction of Refractive Index for Ridge-Shaped Profiles}

The problem of reconstructing the $n_{2}(x)$ profile for layers with a ridge-shaped profile will be considered in this section. The following parabolic distribution will be used:

$$
\begin{array}{r}
n_{2}(x)=-\frac{4\left(n_{2}(L)-n_{2}(0)\right)}{L^{2}}\left(x-\frac{L}{2}\right)^{2}+n_{2}(L), \\
0<x<L .
\end{array}
$$

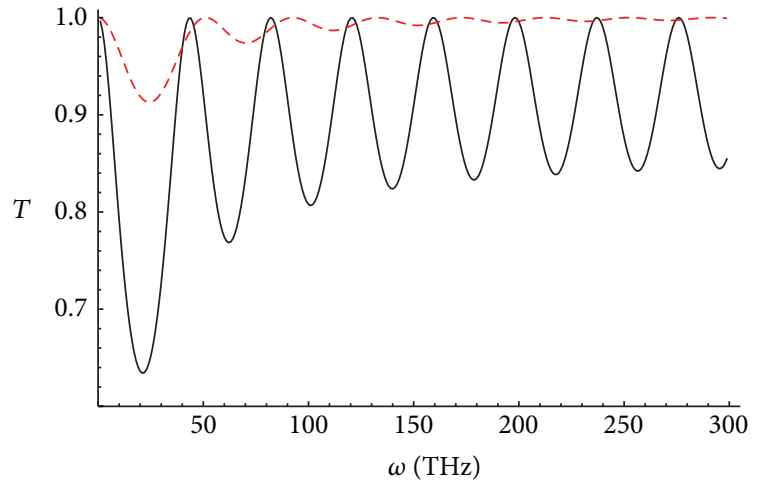

FIgure 2: The layer's transmittance $T$ versus frequency $\omega$. The solid curve corresponds to the discontinuous refractive index, and the red dashed curve is for the continuous refractive index.

In [9], it was shown that layers with different types of gradient filling (linear, sinusoidal, exponential, and logarithmic) have similar amplitude-frequency characteristics differing from each other quite insignificantly.

The layer thickness will be taken as $L=2 \mu \mathrm{m}$. The substance filling the layer will be treated as a composite material defined by the formula $\left(\mathrm{TiO}_{2}\right)_{\chi}\left(\mathrm{SiO}_{2}\right)_{1-\chi}$. Thus, by varying the parameter $\chi$ from 0 to 1 , refractive index will change in the range from 1.5 to 2.1. In our functional, all the values of regularization parameters are taken as zero.

In case of refractive index changing continuously in the whole domain, transited energy has a unique and clearly pronounced minimum. For instance, for a parabolic profile the minimum is close to $24 \mathrm{THz}$ (the dashed line in Figure 2). If refractive index has a leap at both interfaces of the layer, the curve of transited energy will have multiple extrema. For the parabolic profile, this case corresponds to the solid line in Figure 2.

In reconstructing a profile of the dielectric layer's refractive index, it is often quite difficult to take a wide frequency range because of a spread in values $n=n(\omega)$ for various $\omega$. Hence, it is often more convenient to use a narrow frequency interval in which $n(\omega)$ is more or less constant. Note that in our studies absorption caused by wave passage through the layer is not accounted for. More detailed information related to absorption coefficients for a small content of $\mathrm{TiO}_{2}$ can be found in [34].

Profiles reconstructed at various frequency ranges are given in Figure 3. For all cases, the number of iterations $M=$ 50. The best approximation $\varepsilon \approx 3 \cdot 10^{-4}$ is reached at frequencies $23-25 \mathrm{THz}$ (the red dashed curve). The profile corresponding to the red dashed line in Figure 3 is constructed for $50 \mathrm{THz} \leq \omega \leq 52 \mathrm{THz}$. For this case, an error of approximation becomes worse by one order of magnitude and equals to $\varepsilon \approx 2.2 \cdot 10^{-3}$. Finally, the worst approximation $\varepsilon \approx 6.8 \cdot 10^{-3}$ occurs at the range $70-72 \mathrm{THz}$ (the blue dashed-dotted line).

Dependence of an error of the profile reconstruction $\varepsilon$ on the number of iterations $M$ will be considered next. One can apparently expect that while $M$ increases up to a certain value, the error should decrease. From Figures 4 and 5, it is clearly seen that the above expectations are totally met. In the test, 


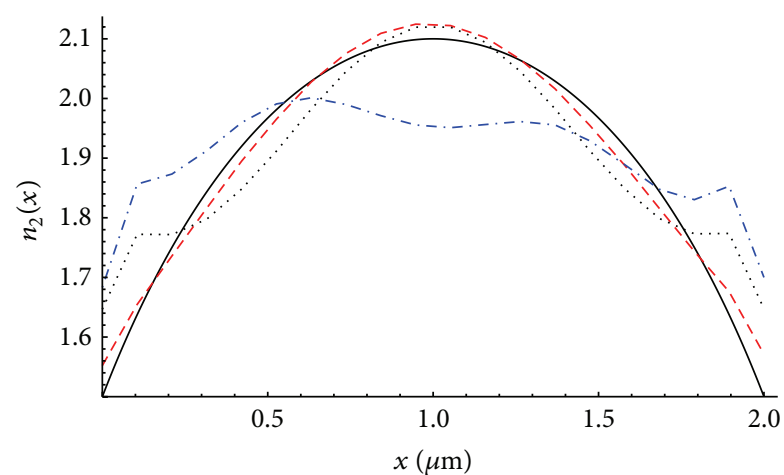

FIGURE 3: Reconstructed profiles. The solid curve corresponds to the initial profile $n_{2}$. The red dashed line corresponds to the reconstructed profile at frequencies $23-25 \mathrm{THz}$, the dotted line corresponds to $50-52 \mathrm{THz}$, and the blue dash-dotted line corresponds to $70-72 \mathrm{THz}$.

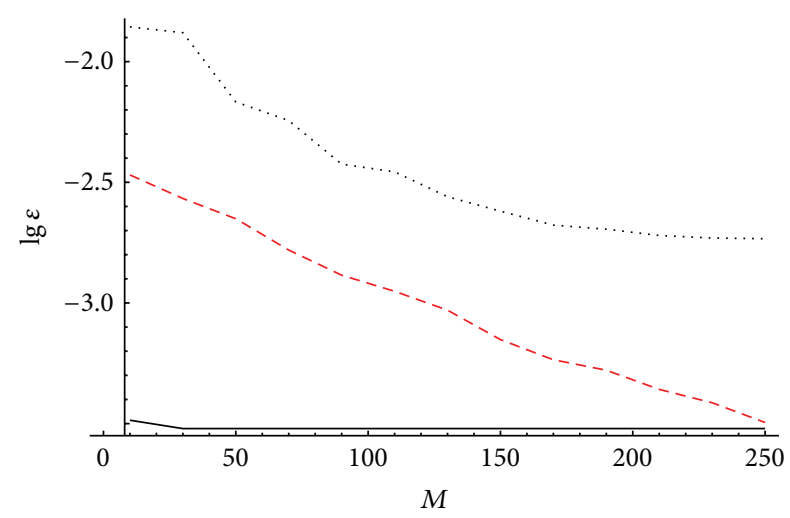

FIGURE 4: The relative error of reconstruction of $n_{2}(x)$ for the parabolic layer versus the number of iterations $M$. Refractive index is continuous in the whole domain. The solid curve corresponds to reconstructing frequencies $23-25 \mathrm{THz}$, the red dashed curve corresponds to reconstructing frequencies $50-52 \mathrm{THz}$, and the dotted curve corresponds to reconstructing frequencies $70-72 \mathrm{THz}$.

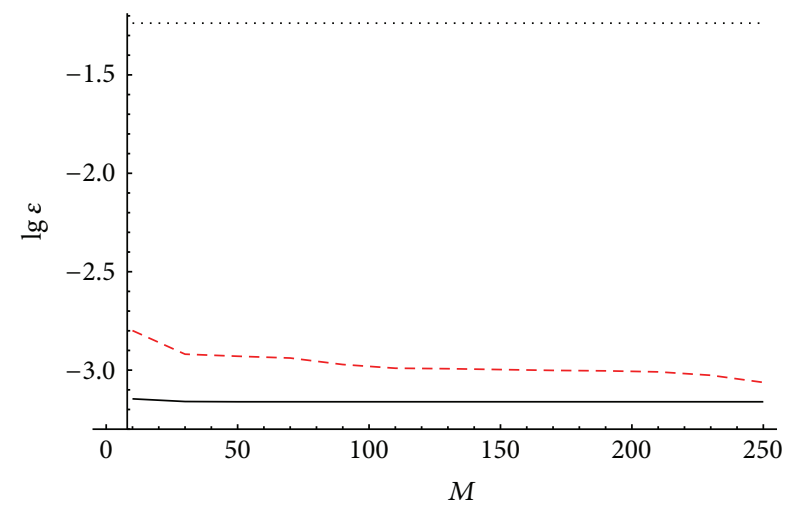

FIGURE 5: The relative error of reconstruction of refractive index for the parabolic layer versus the number of iterations $M$. Refractive index is discontinuous on the layer boundaries. The solid curve corresponds to reconstructing frequencies $20-22 \mathrm{THz}$, the red dashed curve corresponds to reconstructing frequencies $42-44 \mathrm{THz}$, and the dotted curve corresponds to reconstructing frequencies 61$63 \mathrm{THz}$. the number of nodes $N=20$. Graphs showing dependence of lge on $M$ for the parabolic profile are given in Figure 4. Intervals having width of $2 \mathrm{THz}$ are chosen for the reconstruction intervals. The first interval $23-25 \mathrm{THz}$ corresponds to the global minimum of energy transited through the layer. The solid line in Figure 4 depicts the error distribution for this case. The minimum value $\varepsilon \approx 3 \cdot 10^{-3}$ is reached at $M=30$; for a further increase in $M$, the error will no longer depend on $M$.

The second interval for reconstructing the $n_{2}(x)$ profile is $50-52 \mathrm{THz}$. This interval corresponds to the first maximum of the transited energy. In this case, the error decreases slowly with $M$ reaching the optimal value $M=250$. At last, the third interval is $70-72 \mathrm{THz}$. It corresponds to the second minimum of the transited energy (see Figure 2). In this case, the error also decreases with increase in $M$. However, accuracy of the profile reconstruction is worse by one order of magnitude.

Details of the profile reconstruction for the case of $n_{2}(x)$ changing sharply (in leaps) at the layer boundaries will be considered next. In this case, for frequencies of the main minimum of the transited energy the optimal profile is reached at $M<30$ (the solid curve in Figure 5). The frequency interval $42-44 \mathrm{THz}$ also provides a good approximation (the red dashed curve). For this interval, the minimum error is reached at $M=470$ and coincides with the reconstruction error for $\omega \approx 20-22 \mathrm{THz}$. The interval corresponding to the second minimum of transited energy (61-63 THz) practically does not depend on $M$ (the dotted line). The error $\varepsilon$ for the last interval is worse by more than one order of magnitude than the reconstruction errors for the remaining two intervals.

The main conclusion regarding reconstruction of refractive index for ridge-shaped profiles is the following. The optimal frequency interval ensuring the highest accuracy and the minimum number of iterations corresponds to the global minimum of the transited energy. At small shifts from the optimal frequencies, the same error of the profile reconstruction can be reached but at a greater number of iterations.

It is also worth noting that the number of measured values in the optimal frequency range (minimum of the transited energy) is not a key issue at all. The case $J=1$ provides a similar error of approximation of the required profile as the case $J>1$.

\section{Reconstruction of Complex Profiles of Refractive Index}

In this section, reconstruction of more complex profiles will be considered. In Figure 6, the original profile $n_{2}(x)$ is depicted by a solid line. Under the term "complex profile" a profile corresponding to the complex curved geometry is implied (e.g., a case, in which a curve has multiple extremum points). The remaining lines in the figure correspond to profiles reconstructed at various frequency intervals. The red dashed line corresponds to the profile reconstructed at frequencies $65-67 \mathrm{THz}$, the dotted line corresponds to that at frequencies $36-38 \mathrm{THz}$, and the blue dotted line corresponds to that at frequencies $89-91 \mathrm{THz}$.

The frequency interval $65-67 \mathrm{THz}$ corresponds to the minimum of energy transited through the layer (Figure 7). 


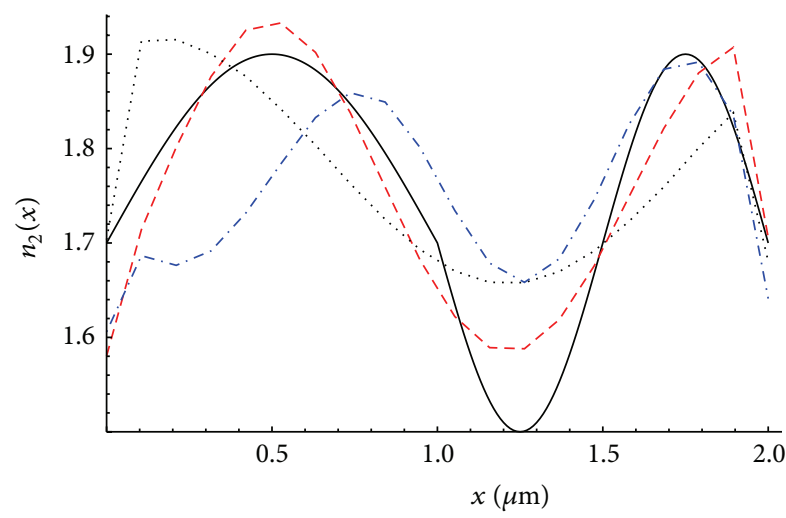

FIGURE 6: Reconstructed profiles. The solid curve corresponds to the initial profile $n_{2}$. The red dashed line corresponds to the reconstructed profile at frequencies $65-67 \mathrm{THz}$, the dotted line corresponds to $36-38 \mathrm{THz}$, and the blue dash-dotted line corresponds to $89-91 \mathrm{THz}$

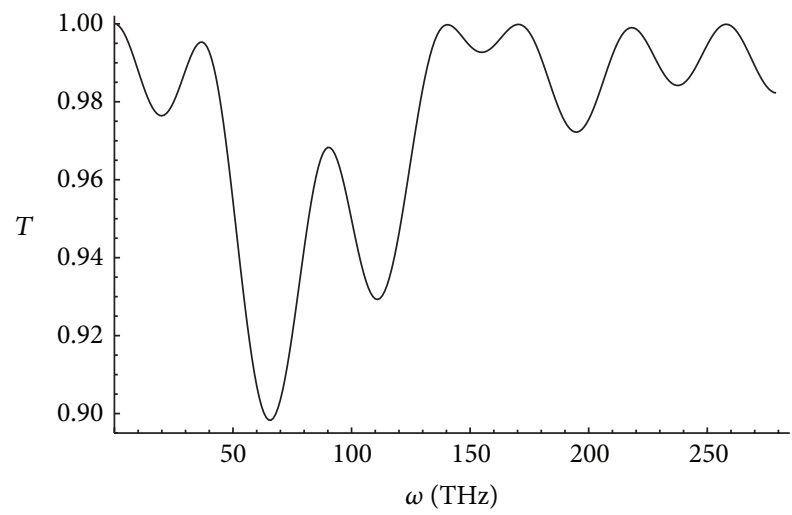

FIgure 7: The layer's transmittance $T$ versus frequency $\omega$. Refractive index is discontinuous.

As in the case of layers having a ridge-shaped profile, this frequency interval can be considered as optimal. It provides the best approximation to the required profile (see Figure 6).

The general behavior of dependence of the approximation error $\varepsilon$ on the number of iterations $M$ remains similar to that for simple profiles. The reconstruction error at $\omega \in$ $(65 \mathrm{THz}, 67 \mathrm{THz})$ will also be minimum (the solid curve in Figure 8). If the frequency interval is shifted from the optimal frequency interval, the approximation error will be worsened (the dashed and dotted lines in Figure 8).

Unlike the previous section, a case of discontinuous refractive index $\left(n_{1}=n_{3}=1.5\right)$ is the only case considered here. It can be noted that the obtained results will remain similar for any choice of values of $n_{1}$ and $n_{3}$. The main conclusion for these types of complex profiles is the same as for the ridgeshaped profiles: the best approximation is reached at the interval in which the transited wave's energy has a minimum.

Just as in the case of simple profiles, the number of measurements in the optimal range for complex profiles is not a key issue at all. A measurement at a single frequency provides the same approximation as measurements carried out at several frequencies.

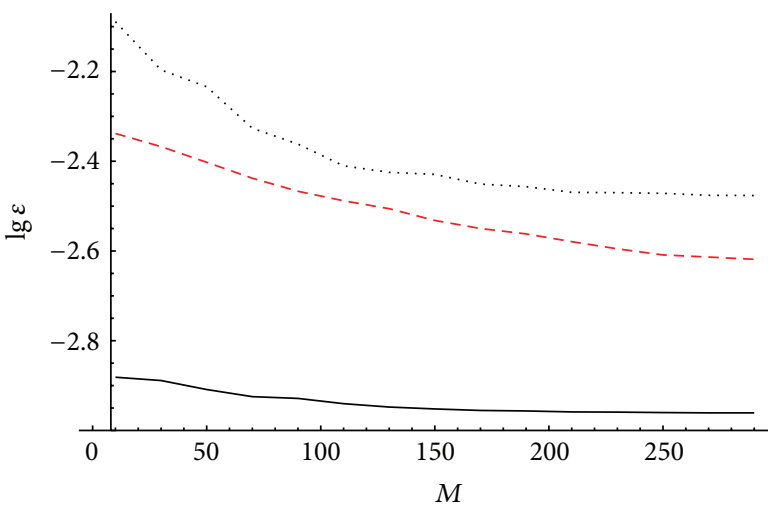

FIGURE 8: The relative error of reconstruction of $n_{2}(x)$ for the parabolic layer versus the number of iterations $M$. Refractive index is continuous in the whole domain. The solid curve corresponds to reconstructing frequencies $65-67 \mathrm{THz}$, the red dashed curve corresponds to reconstructing frequencies $36-38 \mathrm{THz}$, and the dotted curve corresponds to reconstructing frequencies $89-91 \mathrm{THz}$.

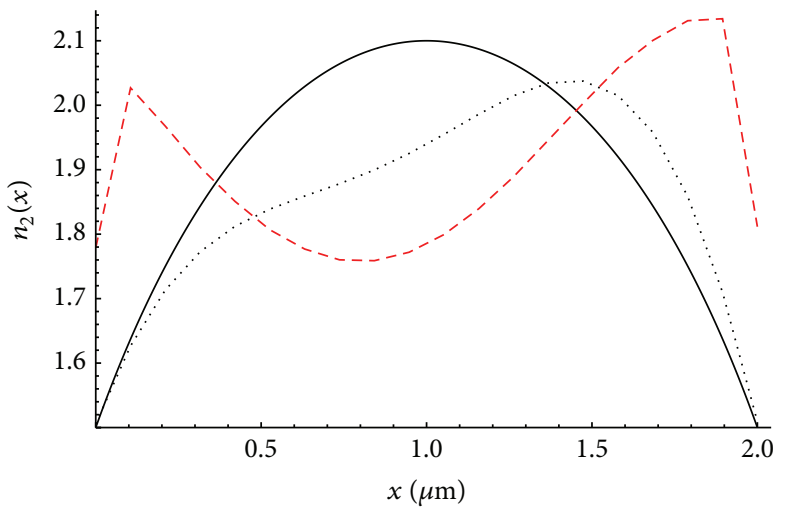

FIGURE 9: Reconstructed profiles for the case of perturbed data $(\delta=$ 0.3 ). The solid curve corresponds to the initial profile $n_{2}$. The red dashed line corresponds to the reconstructed profile at $\alpha=0.2, \beta_{i}=$ 5 , and the dotted line corresponds to $\alpha=\beta_{i}=0$.

\section{Influence of a Measurement Error on the Profile Reconstruction}

In this section, influence of the measurement error $\delta$ on accuracy of the profile reconstruction will be considered. Here the measurement error must be understood as a perturbation of the original data of the following type:

$$
y=y+\delta q y,
$$

where $q$ is a random number, $|q| \leq 1$. Data generated in the above manner will always alter at a new generation.

It certainly makes more sense to perform reconstructions based on several measurements instead of using only one measurement. The measurements can be carried out both at one frequency and at several frequencies. If the measurements undergo perturbations, the inverse problem gets worsened. In this case, regularization parameters must be chosen as nonzero. In Figure 9, results of reconstruction of the parabolic profile described in Section 5 are presented. 
The reconstruction is carried out at three measurements in the vicinity of the minimum $T$. The dashed line corresponds to optimal regularization parameters: $\alpha=0.2$ and $\beta_{i}=5$. For this case, $\varepsilon \approx 0.002$. The dotted line corresponds to reconstruction using the functional with zero regularization parameters $(\varepsilon \approx 0.013)$.

In reconstructing the layer profile using some perturbed data, the optimal interval for reconstruction remains to be the range corresponding to the minimum values of $T$. Unlike the case of accurately measured data in which it is more convenient to take regularization parameters equal to zero, in this case, the functional must be regularized.

\section{Conclusions}

For reconstruction of refractive index, the best results are obtained in cases in which measurements are carried out at frequencies close to frequencies of minimum transition of energy through the layer. The frequency interval can be very narrow such that dispersion effects of the substance filling the layer remain insignificant. The measurements can be also carried out at a single frequency. In this case, accuracy of the profile reconstruction practically remains the same.

Thus, the following strategy can be utilized. First, the flow of energy passed through the layer must be measured. Next, a frequency, at which the flow has a minimum, is selected. High precision measurements for the entire diffracted field at the selected frequency (or at a narrow frequency interval) are carried out at the next stage.

The functional used for the profile reconstruction is constructed with zero regularization parameters. However, if it is known a priori that the measurements were carried out with essential errors, the regularization parameters $\alpha$ and $\beta_{i}$ must be chosen as nonzero.

\section{Conflict of Interests}

The author declares that there is no conflict of interests regarding the publication of this paper.

\section{Acknowledgment}

This work was funded by the subsidy allocated to Kazan Federal University for the state assignment in the sphere of scientific activities.

\section{References}

[1] W. H. Southwell, "Gradient-index antireflection coatings," Optics Letters, vol. 8, no. 11, pp. 584-586, 1983.

[2] J.-C. Zhang, L.-M. Xiong, M. Fang, and H.-B. He, "Wide-angle and broadband gradedrefractive-index antireflection coatings," Chinese Physics B, vol. 22, no. 4, Article ID 044201, 2013.

[3] A. M. Emad, E. A. Hashish, and M. I. Hassan, "Inversion of lossy dielectric profiles using particle swarm optimization," Progress In Electromagnetics Research M, vol. 9, pp. 93-105, 2009.

[4] L. Li and F. Li, "Closed time-domain solutions for 1D scattering and inverse scattering in anisotropic medium," IEEE Transactions on Antennas and Propagation, vol. 56, no. 7, pp. 2061-2066, 2008.
[5] M.-U. Rahman and R. Marklein, "Modeling and inversion of 1-D profiles in time-domain," COMPEL-The International Journal for Computation and Mathematics in Electrical and Electronic Engineering, vol. 28, no. 5, pp. 1349-1361, 2009.

[6] K. I. Zaytsev, V. E. Karasik, I. N. Fokina, and V. I. Alekhnovich, "Invariant embedding technique for medium permittivity profile reconstruction using terahertz time-domain spectroscopy," Optical Engineering, vol. 52, no. 6, Article ID 068203, 2013.

[7] K. I. Zaytsev, N. V. Chernomyrdin, and V. I. Alekhnovich, "Novel technique for medium permittivity profile reconstruction using THz pulsed spectroscopy," Journal of Physics: Conference Series, vol. 486, no. 1, Article ID 012010, 2014.

[8] A. Semnani and M. Kamyab, "An enhanced hybrid method for solving inverse scattering problems," IEEE Transactions on Magnetics, vol. 45, no. 3, pp. 1534-1537, 2009.

[9] N. B. Pleshchinskii and D. N. Tumakov, "Analysis of electromagnetic wave propagation through a layer with graded-index distribution of refraction index," in Proceedings of the Progress in Electromagnetics Research Symposium (PIERS '12), pp. 425-429, Moscow, Russia, August 2012.

[10] Z.-W. Lin, X. Xu, X.-J. Zhang, and G.-Y. Fang, "An inverse electromagnetic scattering method for one-dimensional inhomogeneous media," Chinese Physics Letters, vol. 28, no. 1, Article ID 014101, 2011.

[11] F. H. Ahmad, R. M. Castellane, and E. L. Miller, “Technique for evaluation of profiles of a composite chiral slab through inversion and pseudospectral approximation," IEEE Transactions on Antennas and Propagation, vol. 54, no. 6, pp. 1709-1717, 2006.

[12] A. Casagranda, D. Franceschini, A. Massa, P. M. van den Berg, A. Abubakar, and T. M. Habashy, "The multi-frequency diagonalized contrast source method for electromagnetic inversion," in Proceedings of the European Conference on Antennas and Propagation (EuCAP '06), pp. 1-5, Nice, France, November 2006.

[13] I. C. Mertzanides, G. N. Tsokas, and J. N. Sahalos, "An analytical method for determining the characteristics of two-layered media," in Proceedings of the 2nd International Symposium on Trans Black Sea Region on Applied Electromagnetism, p. 120, June 2000.

[14] E. A. Hashish, "Forward and inverse scattering from an inhomogeneous dielectric slab," Journal of Electromagnetic Waves and Applications, vol. 17, no. 5, pp. 719-736, 2003.

[15] G. Caviglia and A. Morro, "Inversion of reflection data in an isotropic multilayered medium," Acta Mechanica, vol. 189, no. 1-4, pp. 65-72, 2007.

[16] M. Nakhkash, Y. Huang, and M. T. C. Fang, "Application of the multilevel single-linkage method to one-dimensional electromagnetic inverse scattering problem," IEEE Transactions on Antennas and Propagation, vol. 47, no. 11, pp. 1658-1668, 1999.

[17] A. Abubakar, T. M. Habashy, V. L. Druskin, and L. Knizhnerman, "An enhanced Gauss-Newton inversion algorithm using a dual-optimal grid approach," IEEE Transactions on Geoscience and Remote Sensing, vol. 44, no. 6, pp. 1419-1427, 2006.

[18] E. Haber, D. W. Oldenburg, and R. Shekhtman, "Inversion of time domain three-dimensional electromagnetic data," Geophysical Journal International, vol. 171, no. 2, pp. 550-564, 2007.

[19] A. V. Brovko, E. K. Murphy, and V. V. Yakovlev, "A neural network technique for reconstruction of $2 \mathrm{D}$ complex permittivity profiles of materials in waveguide systems," in Proceedings of the Global Congress on Microwave Energy Applications, pp. 381-384, Otsu, Japan, August 2008. 
[20] S. Caorsi, A. Costa, and M. Pastorino, "Microwave imaging within the second-order born approximation: stochastic optimization by a genetic algorithm," IEEE Transactions on Antennas and Propagation, vol. 49, no. 1, pp. 22-31, 2001.

[21] C.-C. Chiu and W.-T. Chen, "ElectromagneticImaging for an imperfectly conducting cylinder by the genetic algorithm," IEEE Transactions on Microwave Theory and Techniques, vol. 48, no. 1, pp. 1901-1905, 2000.

[22] M. Donelli, D. Franceschini, P. Rocca, and A. Massa, "Threedimensional microwave imaging problems solved through an efficient multiscaling particle swarm optimization," IEEE Transactions on Geoscience and Remote Sensing, vol. 47, no. 5, pp. 1467-1481, 2009.

[23] T. Huang and A. S. Mohan, "Application of particle swarm optimization for microwave imaging of lossy dielectric objects," in Proceedings of the IEEE Antennas and Propagation Society International Symposium and USNC/URSI Meeting, vol. 1B, pp. 852-855, Washington, DC, USA, July 2005.

[24] C.-H. Huang, C.-C. Chiu, C.-L. Li, and K.-C. Chen, "Time domain inverse scattering of a two-dimensional homogenous dielectric object with arbitrary shape by particle swarm optimization," Progress in Electromagnetics Research, vol. 82, pp. 381-400, 2008.

[25] A. M. Emad and E. A. Hashish, "One dimensional electromagnetic inversion using particle swarm optimization," in Proceedings of the 24th National Radio Science Conference, vol. B17, pp. 1-8, March 2007.

[26] E. D. Ülker and S. Ülker, "Application of particle swarm optimization to microwave tapered microstrip lines," Computer Science \& Engineering, vol. 4, no. 1, pp. 59-64, 2014.

[27] F. van den Bergh and A. P. Engelbrecht, "A study of particle swarm optimization particle trajectories," Information Sciences, vol. 176, no. 8, pp. 937-971, 2006.

[28] Y. R. Samii, "Genetic algorithms (GA) and particle swarm optimization (PSO) in engineering electromagnetics," in Proceedings of the International Conference on Applied Electromagnetics and Communications (ICECom '03), pp. 1-5, Dubrovnik, Croatia, October 2003.

[29] R. C. Eberhart and Y. H. Shi, "Comparison between genetic algorithms and particle swarm optimization," in Proceedings of the 7 th International Conference on Evolutionary Programming, pp. 611-616, 1998.

[30] B. Wei, E. Şimşek, C. Yu, and Q. H. Liu, "Three-dimensional electromagnetic nonlinear inversion in layered media by a hybrid diagonal tensor approximation: stabilized biconjugate gradient fast Fourier transform method," Waves in Random and Complex Media, vol. 17, no. 2, pp. 129-147, 2007.

[31] G. Franceschini, D. Franceschini, M. Donelli, R. Azaro, and A. Massa, "Electromagnetic inversion of amplitude-only data through a two-step strategy," in Proceedings of the European Conference on Antennas and Propagation (EuCAP '06), Nice, France, November 2006.

[32] N. B. Pleshchinskii and D. N. Tumakov, "The reconstruction of dielectric profile of a layer for the harmonic wave case," in Proceedings of the PIERS 2013, pp. 643-647, Stockholm, Sweden, August 2013.

[33] A. Anufrieva and D. Tumakov, "Peculiarities of electromagnetic wave propagation through layers with ridge-shaped refractive index distribution," in Proceedings of the International Conference on Mathematical Methods in Electromagnetic Theory (MMET '12), pp. 386-389, Kharkiv, Ukraine, August 2012.
[34] D. Y. Smith, C. E. Black, C. C. Homes, and E. Shiles, "Optical properties of $\mathrm{TiO}_{2}-\mathrm{SiO}_{2}$ glass over a wide spectral range," Physica Status Solidi (C) Current Topics in Solid State Physics, vol. 4, no. 3, pp. 838-842, 2007.

[35] A. N. Noemaun, F. W. Mont, J. Cho, E. F. Schubert, G. B. Kim, and C. Sone, "Inductively coupled plasma etching of gradedrefractive-index layers of $\mathrm{TiO}_{2}$ and $\mathrm{SiO}_{2}$ using an ITO hard mask," Journal of Vacuum Science and Technology A: Vacuum, Surfaces and Films, vol. 29, no. 5, Article ID 051302, 2011.

[36] N. B. Pleshchinskii, I. E. Pleshchinskaya, and E. M. Karchevskiy, "The over-determined boundary value problem method in the electromagnetic waves propagation and diffraction theory," in Proceedings of the Progress in Electromagnetics Research Symposium (PIERS '09), pp. 132-136, Moscow, Russia, August 2009.

[37] A. V. Anufrieva, V. L. Kipot, and D. N. Tumakov, "Elastic wave propagation through a layer with graded-index distribution of density," in Proceedings of the Days on Diffraction Conference, pp. 21-26, St. Petersburg, Russia, May-June 2012. 

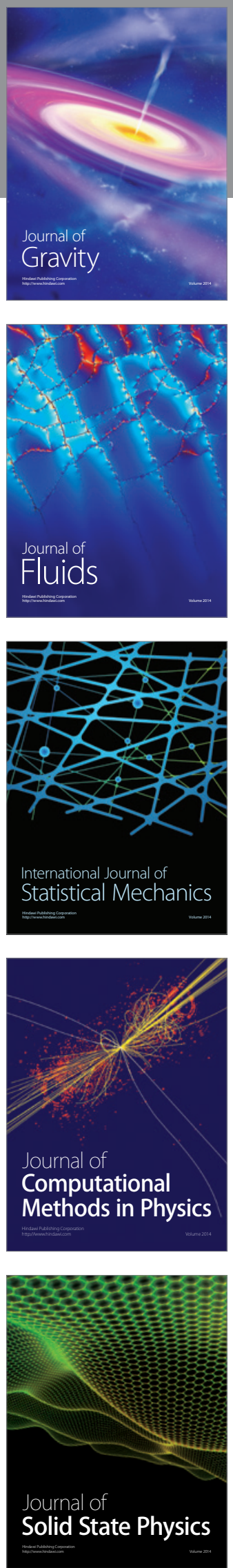

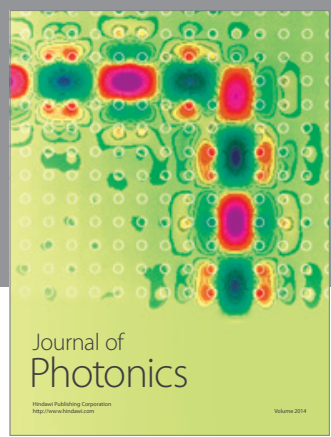

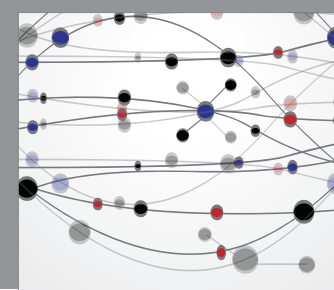

The Scientific World Journal

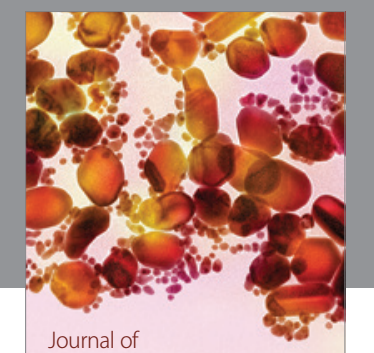

Soft Matter
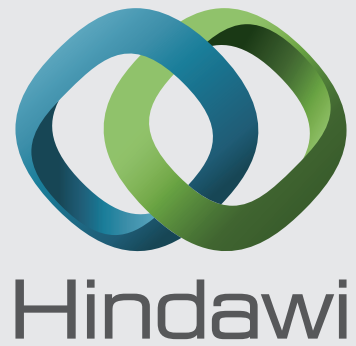

Submit your manuscripts at

http://www.hindawi.com
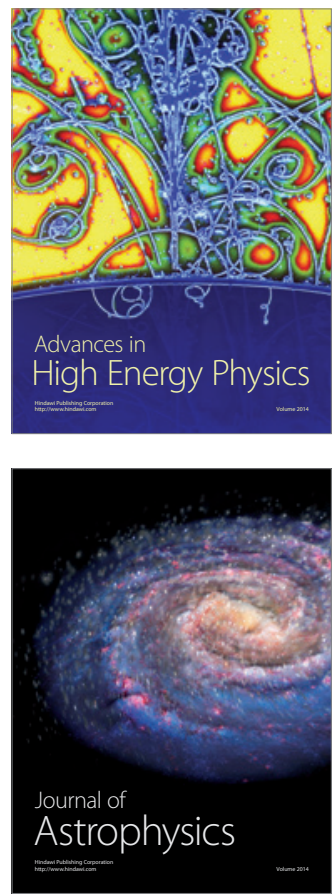
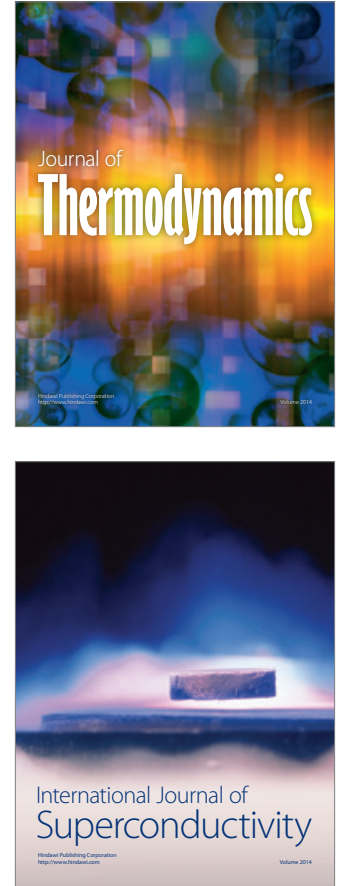
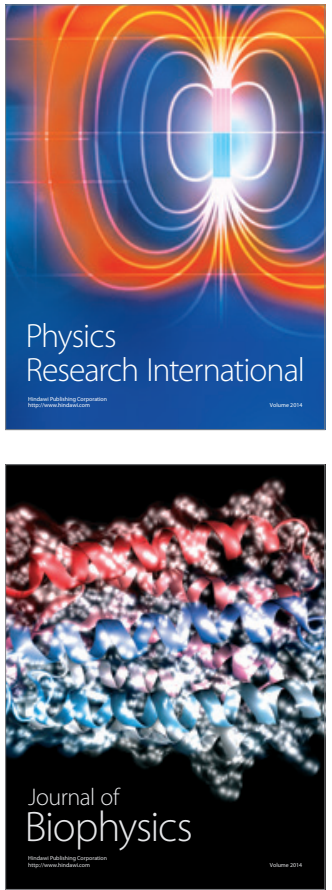
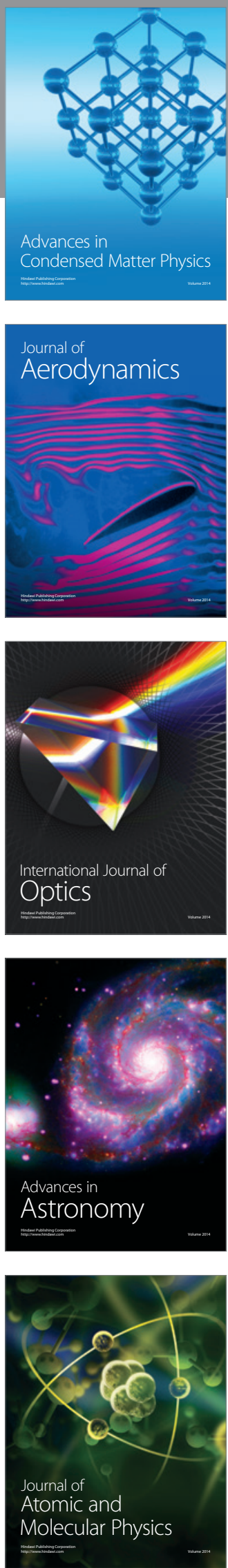